\title{
Utility of intraoperative monitoring with motor-evoked potential during the surgical enucleation of peripheral nerve schwannoma
}

\author{
HIROMI SASAKI $^{1 *}$, SATOSHI NAGANO ${ }^{1 *}$, MASAHIRO YOKOUCHI $^{1}$, TAKAO SETOGUCHI ${ }^{2}$, \\ HIROFUMI SHIMADA ${ }^{1}$, TAKUYA YAMAMOTO ${ }^{1}$, YASUHIRO ISHIDOU ${ }^{3}$ and SETSURO KOMIYA $^{1}$ \\ ${ }^{1}$ Department of Orthopedic Surgery, ${ }^{2}$ The Near-Future Locomotor Organ Medicine Creation Course and \\ ${ }^{3}$ Department of Medical Joint Materials, Graduate School of Medical and Dental Sciences, \\ Kagoshima University, Kagoshima 890-8520, Japan
}

Received October 17, 2015; Accepted February 17, 2017

DOI: $10.3892 / \mathrm{ol} .2018 .8456$

\begin{abstract}
Although it is thought that the surgical enucleation of schwannomas can be easily performed, certain patients present with postoperative neurological symptoms. The present study examined the utility of intraoperative motor-evoked potential (MEP) in predicting neurological deficits following the surgical enucleation of peripheral nerve schwannoma. The current study included 23 patients and MEP was performed using transcranial electrical stimulation. In three cases, the MEP decreased to $<50 \%$ of the preoperative value; however, in two cases that involved the peroneal nerve and tibial nerve, results appeared to be false positives induced by a tourniquet during surgery. In another case, the MEP was completely lost following enucleation of the tumor from the sciatic nerve, which recovered to $61 \%$ of the original MEP within $10 \mathrm{~min}$. This patient presented with common peroneal palsy postoperatively. By contrast, another case involving the lumbar nerve root and in which there was reversible postoperative motor loss, the MEP did not change intraoperatively. Postoperative neurological deficit occurred in $22 \%$ of patients in the present study, which is similar to that of previous reports. The present study also demonstrated that even if a nerve is not transected or injured, traction or compression of a peripheral nerve may induce ischemia, which can be monitored using MEP. Although MEP alone was not able to predict postoperative transient sensory or motor deficits, the combination of MEP with other methods of neurological
\end{abstract}

Correspondence to: Dr Satoshi Nagano, Department of Orthopedic Surgery, Graduate School of Medical and Dental Sciences, Kagoshima University, 8-35-1 Sakuragaoka, Kagoshima 890-8520, Japan

E-mail: naga@m2.kufm.kagoshima-u.ac.jp

*Contributed equally

Key words: schwannoma, enucleation, neurological deficit, motor-evoked potential, ischemia, monitoring monitoring may improve accuracy and should be investigated in future studies.

\section{Introduction}

Schwannoma is a relatively rare tumor, comprising 5\% of benign soft tissue tumors (1). Compared with other benign neurogenic tumors, schwannoma originates from a nerve sheath, whereas neurofibroma arises from the nerve itself. Because schwannomas are typically well encapsulated and displace nerve fibers as they grow, it is thought that enucleation can easily be performed without causing postoperative neurological deficit. However, in certain cases it is difficult to enucleate the tumor without causing nerve damage, even when using meticulous operative techniques to preserve nerve fascicles, and such patients may present with postoperative neurological symptoms. The reported incidence of postoperative neurological deficit resulting from the enucleation of schwannoma varies; notably, Park et al (2) reported an incidence of $\leq 75 \%$ in upper-extremity schwannoma. In addition, an association was identified between the presence of Tinel's sign and increased tumor volume with increased risk of postoperative nerve injury (2). One method for monitoring postoperative neurologic symptoms is intraoperative motor-evoked potential (MEP). MEP was originally developed as a method for monitoring cranial nerve function during cerebral aneurysm surgery and brain tumor resection; however, it is now widely applied in different types of neurosurgery, and MEP monitoring is recommended as a precaution against perioperative neuroparalysis in a spinal cord tumor resection and scoliosis surgery (3-6). However, to the best of our knowledge, no previous studies have attempted to apply MEP for intraoperative neurological monitoring during the enucleation of peripheral nerve schwannoma. The present study examined the utility of MEP in predicting postoperative neurological deficit following the surgical enucleation of schwannoma.

\section{Materials and methods}

Patients. The current study included 23 patients $[9$ male, 14 female; age range, 29-78 years old (mean, 55 years old)] with 
schwannoma of a peripheral nerve excluding a pure sensory nerve, who underwent surgical enucleation at the department of orthopedic surgery in Kagoshima University between 2011 and 2014 (Table I). Preoperative Magnetic Resonance Imaging (MRI) was underwent for identifying the tumor's location in all cases. The most frequently involved nerves were tibial $(n=6)$ and sciatic $(n=5)$. A Tinel's-like sign or paresthesia that was painful to percussion was identified preoperatively in $16 / 21$ cases $(76 \%$; excluding two cases of schwannoma originating from the lumbar nerve root). All procedures were in accordance with the ethical standards of the institutional Review Board of Kagoshima University and with the 1964 Helsinki declaration and its later amendments or comparable ethical standards. The patients were informed that data from the case would be submitted for publication, and gave their consent.

Intraoperative MEP. MEP was performed with transcranial electrical stimulation. The transcranial stimulation was typically delivered in trains of five pulses with $2.0 \mathrm{msec}$ interval at $500 \mathrm{~V}(0.1 \mathrm{~Hz}$ frequency) by stimulator (SEN-4100; Nihon Kohden, Tokyo, Japan). The thenar, flexor carpi ulnaris and brachioradialis muscles were monitored in cases involving upper extremities. In cases associated with lower extremities the anterior tibialis or gastrocnemius muscle were monitored for the peroneal or tibial nerves, respectively. MEP was measured prior to and following surgical enucleation using four-channel electromyography (MEB-9140; Nihon Kohden). A decrease in MEP of $<50 \%$ of the preoperative value was designated as alarm point indicating loss of motor function.

Surgical enucleation. General anesthesia was induced in all cases. Care was taken not to influence the MEP by maintaining narcotic and intravenous anesthesia rather than employing an inhaled anesthetic and muscle relaxant. At the induction of anesthesia, propofol $(0.5 \mathrm{mg} / \mathrm{kg} / 10 \mathrm{sec})$, fentanyl $(1.5 \sim 8 \mu \mathrm{g} / \mathrm{kg})$ and vecuronium $(0.1 \mathrm{mg} / \mathrm{kg})$ were used. Anesthesia was maintained using propofol $(4 \sim 10 \mathrm{mg} / \mathrm{kg} / \mathrm{h})$ and fentanyl $(0.5 \sim 5 \mu \mathrm{g} / \mathrm{kg} / \mathrm{h})$. The surgery began with a longitudinal incision over the tumor, followed by incision of the fascia to expose the tumor. Prior to enucleation of the tumor body, the connecting nerve at the proximal and distal parts of the tumor was identified and dissected. This procedure loosens the involved nerve trunk and decreases the likelihood of nerve injury (Figs. 1D and 2C). Following dissection of the surrounding connective tissue, a longitudinal incision was made on the tumor capsule in a position that would affect the fascicle (Fig. 2D), exposing the yellowish tumor body. Subsequently, a blunt dissection between the capsule and the tumor resulted in en bloc enucleation of the schwannoma with preservation of the nerve fascicles outside of the capsule. For cases in which a tumor located in a limb was identified on preoperative MRI to be close to a major blood vessel, the limb was exsanguinated and a pneumatic tourniquet applied prior to incision.

\section{Results}

In $3 / 23$ cases, MEP decreased to $<50 \%$ of the preoperative value (Table I). In cases no. 4 and 12, the schwannoma occurred in the calf and involved the common peroneal nerve and tibial nerve, respectively. There was no postoperative neurological deficit in either case; however, because a tourniquet was used during surgery, these results were thought to be false positives. In another case where MEP decreased by $<50 \%$ (case no. 21), the tumor originated from the sciatic nerve (Fig. 2A and B). During surgical exposure of the tumor, and the tibial and peroneal nerves, the MEP remained intact (Fig. 2E). Following enucleation of the tumor from the capsule, with preservation of the affected nerve, the potential was completely lost (Fig. 2E). After $10 \mathrm{~min}$, the MEP recovered to $61 \%$ of the preoperative MEP. The patient in this case presented with common peroneal palsy postoperatively (Table I). In another case in which there was postoperative motor loss (case no. 15), the schwannoma involved the lumbar nerve root; however, the MEP did not change intraoperatively (data not shown). The patient had loss of muscle strength around the hip and knee that recovered 3 months following surgery. A total of 3 other patients (case no. 2,9 and 10) had sensory disturbance in the area of the involved nerve (ulnar or peroneal) postoperatively (Table I). The sensory disturbance was transient in all 3 cases and gradually resolved with 1-4 months. Preoperative neurological symptoms were present in 2 cases (case no. 2 and 6). There were no postoperative complications in either case. One patient (no. 2) had sensory disturbance postoperatively described above, and the other (no. 6) had no postoperative complications.

\section{Discussion}

Enucleation of schwannoma is possible because the tumor occurs in a nerve sheath and does not involve the nerve fibers. However, various rates of postoperative neurological deficit have been demonstrated. Artico et al (7), reported that among 73 cases of resectable schwannoma, preoperative symptoms improved in $41 \%$, worsened $6.8 \%$ and remained unchanged in $52 \%$ of patients postoperatively. Oberle et al (8) identified that sensory disturbance occurred immediately following surgery in 6/12 patients. Notably, Donner et al (9) reported that, out of 31 patients with schwannoma who had preoperative muscle weakness, $13 \%$ experienced postoperative loss of muscle strength. In the present study, postoperative neurological deficit occurred in $22 \%$ of patients, which is similar to that of previous reports. The cause of postoperative neurological deficit in schwannoma remains unclear, although several mechanisms have been proposed, including preoperative nerve compression by the tumor, mechanical nerve injury during surgery, or ischemia of the nerve associated with the surgical procedure. Although postoperative neurological symptoms in patients with peripheral schwannoma are transient in the majority cases, it can be a problem in terms of patient satisfaction with the surgery.

Sawada et al (10), reported that 4/17 cases of schwannoma occurring in the limbs were located in the subclavicular area or brachial plexus and could not be enucleated. Due to the anatomical complexity of subclavicular and brachial plexus schwannomas, an adequate operative field may be difficult to secure, which can result in incomplete enucleation and a higher risk of recurrence. In such cases, sufficient exposure of the nerve and tumor should be conducted with a form of nerve monitoring to predict intraoperative nerve injury. In the 
Table I. Clinicopathological characteristics of the 23 patients with peripheral nerve schwannoma who underwent surgical enucleation in the present study.

\begin{tabular}{|c|c|c|c|c|c|c|c|c|}
\hline \multirow[b]{2}{*}{ Case no. } & \multirow{2}{*}{$\begin{array}{l}\text { Age } \\
\text { (years) }\end{array}$} & \multirow[b]{2}{*}{ Sex } & \multirow{2}{*}{$\begin{array}{l}\text { Nerve involved } \\
\text { with the tumor }\end{array}$} & \multirow{2}{*}{$\begin{array}{l}\text { Pneumatic } \\
\text { tourniquet }\end{array}$} & \multirow[b]{2}{*}{ Tinel's sign } & \multicolumn{2}{|c|}{ Neurological symptoms } & \multirow[b]{2}{*}{$\triangle \mathrm{MEP}<50 \%$} \\
\hline & & & & & & Preoperative & Postoperative & \\
\hline 1 & 78 & M & Sciatic & - & - & No & No & - \\
\hline 2 & 55 & $\mathrm{~F}$ & Peroneal & - & + & Hypesthesia & Numbness, pain & - \\
\hline 3 & 68 & M & Tibial & - & + & No & No & - \\
\hline 4 & 49 & $\mathrm{~F}$ & Tibial & + & + & No & No & + \\
\hline 5 & 58 & M & Tibial & - & + & No & No & - \\
\hline 6 & 63 & $\mathrm{~F}$ & Brachial plexus & - & + & Ulnar nerve palsy & No & - \\
\hline 7 & 36 & F & Median & + & + & No & No & - \\
\hline 8 & 50 & F & Femoral & - & + & No & No & - \\
\hline 9 & 61 & M & Ulnar & - & + & No & Hypesthesia & - \\
\hline 10 & 59 & $\mathrm{~F}$ & Peroneal & - & + & No & Hypesthesia & - \\
\hline 11 & 68 & $\mathrm{~F}$ & Femoral & - & - & No & No & - \\
\hline 12 & 50 & M & Tibial & + & - & No & No & + \\
\hline 13 & 58 & $\mathrm{~F}$ & Tibial & - & + & No & No & - \\
\hline 14 & 55 & F & Brachial plexus & - & + & No & No & - \\
\hline 15 & 51 & F & L4 nerve root & - & - & No & Muscle weakness & - \\
\hline 16 & 70 & $\mathrm{~F}$ & L3 nerve root & - & - & No & No & - \\
\hline 17 & 39 & M & Sciatic & - & - & No & No & - \\
\hline 18 & 63 & $\mathrm{~F}$ & Sciatic & - & + & No & No & - \\
\hline 19 & 62 & M & Median & - & + & No & No & - \\
\hline 20 & 29 & $\mathrm{~F}$ & Tibial & - & + & No & No & - \\
\hline 21 & 51 & M & Sciatic & - & + & No & Peroneal nerve palsy & + \\
\hline 22 & 35 & $\mathrm{~F}$ & Median & - & - & No & No & - \\
\hline 23 & 60 & M & Sciatic & - & + & No & No & - \\
\hline
\end{tabular}

M, male; F, female; L, lumbar.

present study, two cases involved the brachial plexus. In one of these cases, the middle part of the clavicle was transected to obtain adequate exposure of the major blood vessels and nerves. Although there is the risk of delayed union of a repositioned clavicle, this was a good option for reducing the risk of vessel or nerve injury in the current study.

Various methods of perioperative neurological monitoring have been developed, including spontaneous electromyography (spEMG) and somatosensory evoked potential (SSEP), in addition to MEP. In a large study of 1,055 patients who underwent cervical spinal surgery, the sensitivities and specificities of spEMG, SSEP, and MEP were 46 and 73\%, 52 and $100 \%$, and 100 and $96 \%$, respectively (11). MEP is recognized as the most useful type of neurological monitoring and is recommended for use alone or in combination with other monitoring modalities, depending on the risks of the surgery being undertaken (11). Therefore, neurological monitoring using MEP has been widely used for brain and spinal surgeries. For example, in corrective surgery for highly deformed spinal columns, the risk of neurological complications can be as high as $27 \%$ (12). To reduce the risk of such complications, the utility of perioperative nerve monitoring with MEP as a predictor of postoperative neurologic deficit has been studied $(13,14)$. The Japanese Society for Spine Surgery and Related Research conducted a multicenter study of intraoperative never monitoring with MEP in 959 spinal surgeries to determine a warning threshold a cut-off percent for the change between pre- and postoperative MEP (15).

Few studies have applied nerve monitoring to surgeries involving peripheral nerves. Several studies used perioperative neurological monitoring of the peripheral nerves to predict cervical spinal nerve 5 (C5) paralysis during cervical spinal surgery $(16,17)$. Jimenez et al $(16)$, reported that perioperative monitoring with spEMG was an effective predictor of C5 paralysis following cervical spinal surgery, and other studies demonstrated similar results $(17,18)$. Bose et al $(18)$, evaluated whether MEP monitoring could predict C5 neuroparalysis by defining a cut-off value as a decrease in MEP of $<50 \%$ of the baseline value. The results revealed that MEP was able to detect C5 paralysis with a sensitivity and specificity of 91 and $89 \%$, respectively. In contrast, the sensitivity and specificity of spEMG (42 and $85 \%)$ and SSEP (0 and 98\%) led to the conclusion that MEP was the most useful form of nerve monitoring. Bhalodia et al (19), examined the ability of SSEP and MEP to predict postoperative C5 paralysis, and reported that it was difficult to predict with either modality, whether 
A

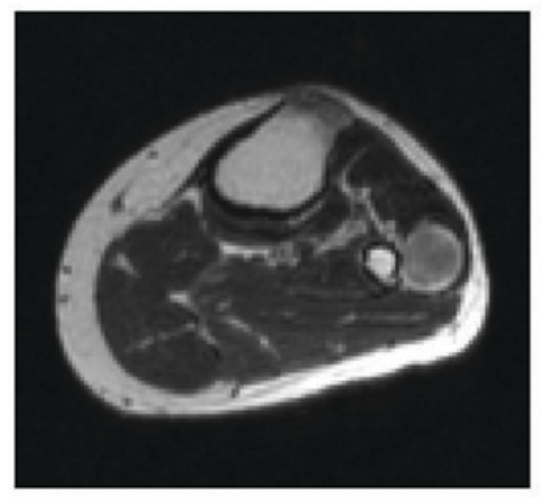

D

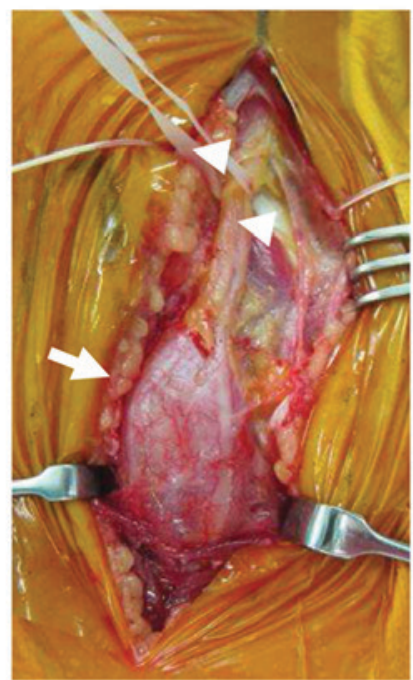

B

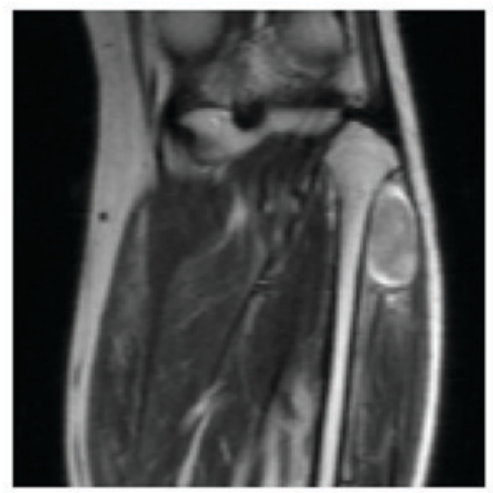

$\mathrm{E}$

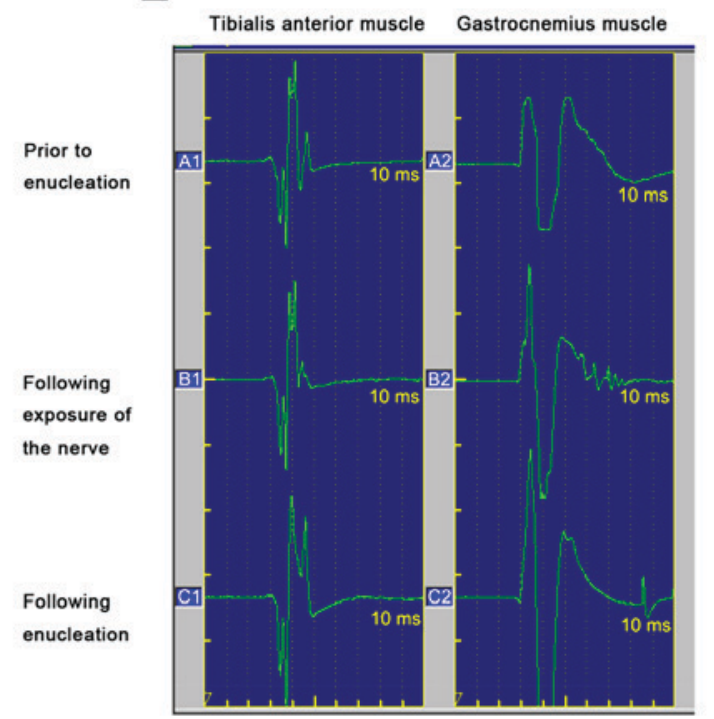

C

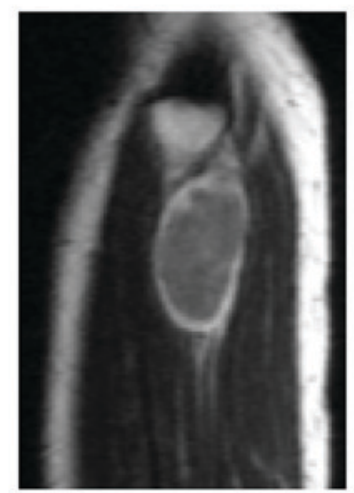

$\mathrm{F}$

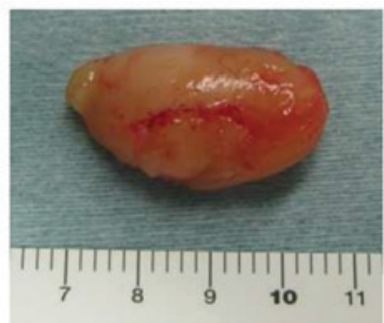

G

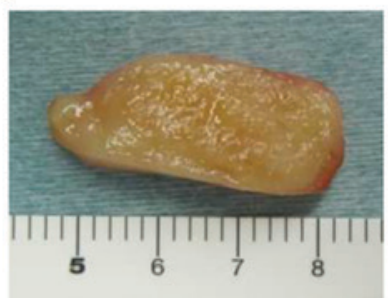

Figure 1. A case of schwannoma arising from the common peroneal nerve (case no. 10). (A) Axial, (B) coronal and (C) sagittal views of T2-weighted magnetic resonance images of the affected area illustrating the target sign, a hyperintense rim and central area of low signal. (D) Intraoperative image illustrating good exposure and preservation of the common peroneal nerve prior to enucleation of the tumor. White arrow heads indicate the sheath of common peroneal nerve, and white arrow indicates the tumor. (E) Motor-evoked potential readings of tibialis anterior muscle (left column) and gastrocnemius muscle (right column) did not decrease notably (top, prior to surgery; middle, following exposure of the nerve; bottom, following enucleation). (F) Macroscopic appearance of the tumor removed and (G) yellowish cut surface.

used alone or in combination. The low sensitivity of SSEP was attributed to the differing structures of cranial and peripheral nerves. Another disadvantage of MEP is that it frequently produces false positive results (17).

No other studies have investigated MEP as a method for monitoring peripheral nerves during the surgical enucleation of schwannoma, to the best of our knowledge. In the present study, there were two cases with false positive results; however, in these cases a pneumatic tourniquet was used intraoperatively. This suggests that the MEP level may depend on blood flow to peripheral nerves. It has previously been reported that MEP is a sensitive indicator of spinal cord ischemia (20). Therefore, even if a nerve is not transected or injured, traction or compression of a peripheral nerve may induce ischemia, which can affect the MEP. In one case in the present study, the MEP suddenly decreased following enucleation of the tumor and the patient developed transient but complete peroneal nerve palsy postoperatively. Although the nerve trunk was preserved, intraoperative ischemia caused by traction or compression of the nerve may have been responsible. This suggests that great care should be taken when preserving the vessels around the nerve and that the MEP should be checked frequently when handling vessels near the schwannoma. Nevertheless, MEP alone was not able to predict postoperative motor loss, suggesting that further combined monitoring with free-run electromyography or direct electrical stimulation (21) may aid in the accurate prediction of nerve injury.

In conclusion, the present study examined the utility of MEP as a perioperative nerve monitoring technique during the enucleation of peripheral nerve schwannomas. Decreased blood flow caused by the pneumatic tourniquet was observed to result in a decrease in MEP. Although MEP alone was not able to predict postoperative transient sensory or motor deficits following the enucleation of schwannoma, the combination of MEP with other methods of neurological monitoring may improve the accuracy of nerve monitoring and should be investigated in future studies. 

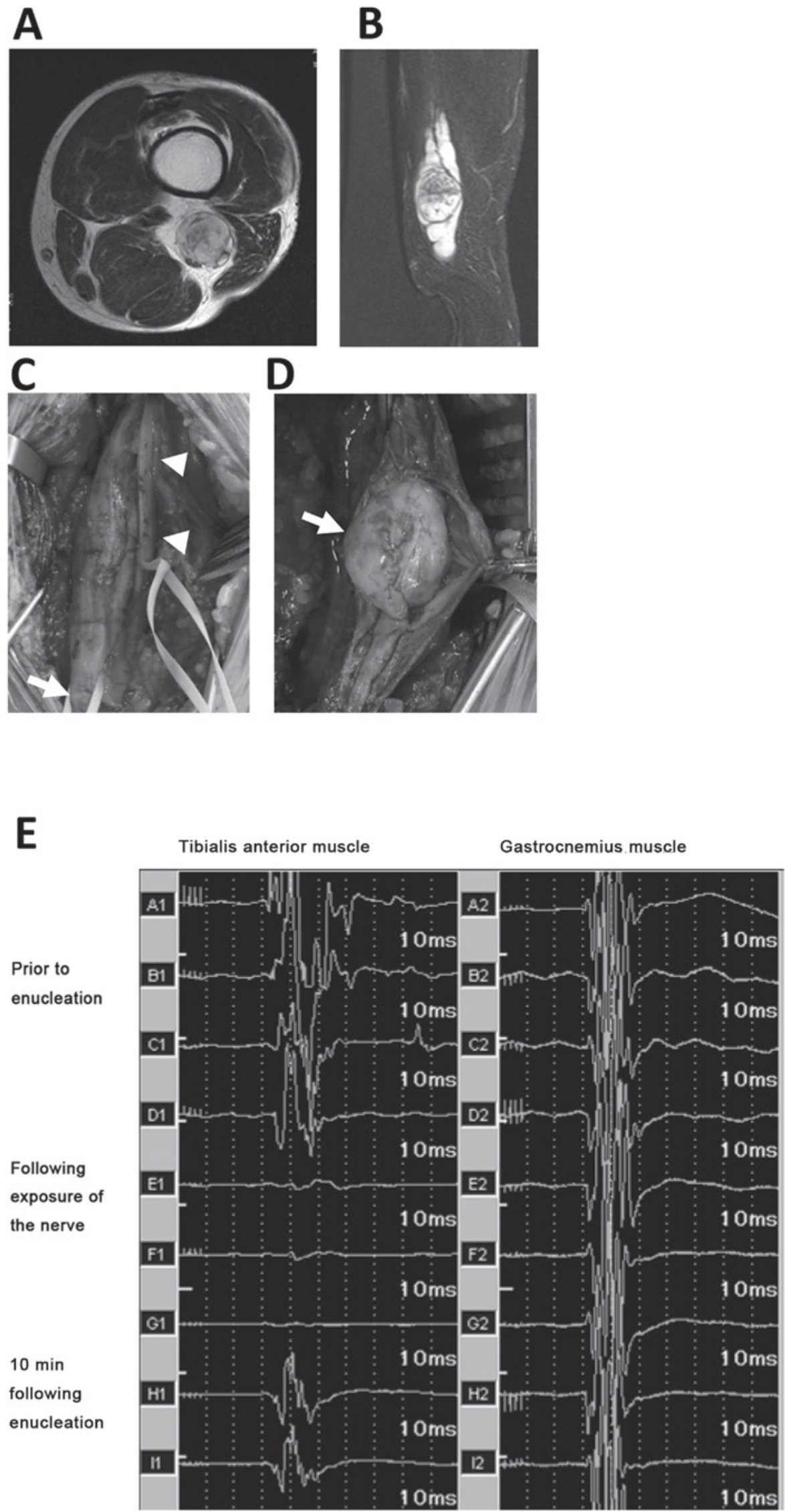

Figure 2. A case of schwannoma affecting the sciatic nerve (case no. 21). (A) Axial and (B) sagittal views of T2-weighted magnetic resonance imaging of the affected area. (C) The tibial (arrowheads) and common peroneal (arrow) nerves were exposed prior to enucleation. (D) The capsule was cut and enucleation performed with the tibial and common peroneal nerves intact. (E) MEP readings of the tibialis anterior muscle (left column) and gastrocnemius muscle (right column) remained intact prior to enucleation (top four waves). Following tumor enucleation, the MEP was completely lost (E1 and F1) but recovered after $10 \mathrm{~min}(\mathrm{H1})$. The patient in this case developed complete peroneal nerve palsy postoperatively. MEP, motor-evoked potential. 


\section{Acknowledgments}

Not applicable.

\section{Competing interests}

The authors declare that they have no competing interests.

\section{References}

1. Kransdorf MJ: Benign soft-tissue tumors in a large referral population: Distribution of specific diagnoses by age, sex, and location. AJR Am J Roentgenol 164: 395-402, 1995.

2. Park MJ, Seo KN and Kang HJ: Neurological deficit after surgical enucleation of schwannomas of the upper limb. J Bone Joint Surg Br 91: 1482-1486, 2009.

3. Hyun SJ and Rhim SC: Combined motor and somatosensory evoked potential monitoring for intramedullary spinal cord tumor surgery: Correlation of clinical and neurophysiological data in 17 consecutive procedures. Br J Neurosurg 23: 393-400, 2009.

4. Epstein NE: The need to add motor evoked potential monitoring to somatosensory and electromyographic monitoring in cervical spine surgery. Surg Neurol Int 4 (Suppl 5): S383-S391, 2013.

5. Feng B, Qiu G, Shen J, Zhang J, Tian Y, Li S, Zhao H and Zhao Y: Impact of multimodal intraoperative monitoring during surgery for spine deformity and potential risk factors for neurological monitoring changes. J Spinal Disord Tech 25: E108-E114, 2012.

6. Pastorelli F, Di Silvestre M, Plasmati R, Michelucci R, Greggi T Morigi A, Bacchin MR, Bonarelli S, Cioni A, Vommaro F, et al: The prevention of neural complications in the surgical treatment of scoliosis: The role of the neurophysiological intraoperative monitoring. Eur Spine J 20 (Suppl 1): S105-S114, 2011.

7. Artico M, Cervoni L, Wierzbicki V, D'Andrea V and Nucci F: Benign neural sheath tumours of major nerves: Characteristics in 119 surgical cases. Acta Neurochir (Wien) 139: 1108-1116, 1997.

8. Oberle J, Kahamba J and Richter HP: Peripheral nerve schwannomas-an analysis of 16 patients. Acta Neurochir (Wien) 139: 949-953, 1997.

9. Donner TR, Voorhies RM and Kline DG: Neural sheath tumors of major nerves. J Neurosurg 81: 362-373, 1994.

10. Sawada T, Sano M, Ogihara H, Omura T, Miura K and Nagano A: The relationship between pre-operative symptoms, operative findings and postoperative complications in schwannomas. J Hand Surg Br 31: 629-634, 2006.

11. Kelleher MO, Tan G, Sarjeant R and Fehlings MG: Predictive value of intraoperative neurophysiological monitoring during cervical spine surgery: A prospective analysis of 1055 consecutive patients. J Neurosurg Spine 8: 215-221, 2008.
12. Lenke LG, Newton PO, Sucato DJ, Shufflebarger HL, Emans JB, Sponseller PD, Shah SA, Sides BA and Blanke KM: Complications after 147 consecutive vertebral column resections for severe pediatric spinal deformity: A multicenter analysis. Spine (Phila Pa 1976) 38: 119-132, 2013.

13. Hilibrand AS, Schwartz DM, Sethuraman V, Vaccaro AR and Albert TJ: Comparison of transcranial electric motor and somatosensory evoked potential monitoring during cervical spine surgery. J Bone Joint Surg Am 86-A: 1248-1253, 2004.

14. Schwartz DM, Sestokas AK, Dormans JP, Vaccaro AR, Hilibrand AS, Flynn JM, Li PM, Shah SA, Welch W, Drummond DS and Albert TJ: Transcranial electric motor evoked potential monitoring during spine surgery: Is it safe? Spine (Phila Pa 1976) 36: 1046-1049, 2011.

15. Kobayashi S, Matsuyama Y, Shinomiya K, Kawabata S, Ando M, Kanchiku T, Saito T, Takahashi M, Ito Z, Muramoto A, et al: A new alarm point of transcranial electrical stimulation motor evoked potentials for intraoperative spinal cord monitoring: A prospective multicenter study from the spinal cord monitoring working group of the Japanese society for spine surgery and related research. J Neurosurg Spine 20: 102-107, 2014.

16. Jimenez JC, Sani S, Braverman B, Deutsch H and Ratliff JK: Palsies of the fifth cervical nerve root after cervical decompression: Prevention using continuous intraoperative electromyography monitoring. J Neurosurg Spine 3: 92-97, 2005.

17. Fan D, Schwartz DM, Vaccaro AR, Hilibrand AS and Albert TJ: Intraoperative neurophysiologic detection of iatrogenic C5 nerve root injury during laminectomy for cervical compression myelopathy. Spine (Phila Pa 1976) 27: 2499-2502, 2002.

18. Bose B, Sestokas AK and Schwartz DM: Neurophysiological detection of iatrogenic $\mathrm{C}-5$ nerve deficit during anterior cervical spinal surgery. J Neurosurg Spine 6: 381-385, 2007.

19. Bhalodia VM, Schwartz DM, Sestokas AK, Bloomgarden G, Arkins T, Tomak P, Gorelick J, Wijesekera S, Beiner J and Goodrich I: Efficacy of intraoperative monitoring of transcranial electrical stimulation-induced motor evoked potentials and spontaneous electromyography activity to identify acute-versus delayed-onset C-5 nerve root palsy during cervical spine surgery: Clinical article. J Neurosurg Spine 19: 395-402, 2013.

20. Kai Y, Owen JH, Allen BT, Dobras M and Davis C: Relationship between evoked potentials and clinical status in spinal cord ischemia. Spine (Phila Pa 1976) 20: 291-296, 1995.

21. Leppanen RE: Intraoperative monitoring of segmental spinal nerve root function with free-run and electrically-triggered electromyography and spinal cord function with reflexes and F-responses. A position statement by the American Society of Neurophysiological Monitoring. J Clin Monit Comput 19: 437-461, 2005

This work is licensed under a Creative Commons Attribution-NonCommercial-NoDerivatives 4.0 International (CC BY-NC-ND 4.0) License. 